\title{
NGC 6388: chemical composition of its 8 cool giants
}

\author{
G. Wallerstein ${ }^{1}$, V. Kovtyukh ${ }^{2}$, and S. Andrievsky ${ }^{2}$ \\ ${ }^{1}$ Department of Astronomy, University of Washington, Seattle, WA 98195 \\ email: wall@astro.washington.edu \\ ${ }^{2}$ Department of Astronomy and Astronomical Observatory, Odessa National University, T.G. \\ Shevchenko Park, 65014, Odessa, Ukraine \\ email: val@deneb.odessa.ua
}

\begin{abstract}
Eight cool giants in the unusual globular cluster NGC 6388 have been investigated in order to derive their elemental abundances. Average relative-to-solar abundance of iron is about -0.8 . We have found that oxygen abundance is reduced as compared to most stars of a similar metallicity. The reduced carbon as compared to oxygen is at the level to be expected for red giant tip stars. Abundance of the rest of investigated elements are within the expected limits for the globular cluster stars.
\end{abstract}

Keywords. Globular clusters: individual (NGC 6388)

\section{Introduction}

NGC 6388 is an unusual cluster in several respects. It is located in the Galactic Bulge. Its red giant branch reaches only to 2 magnitudes above the horizontal branch indicating a rather high metallicity, typical of bulge clusters. In addition its horizontal branch is dominated by red stars, as expected for a relatively metal-rich cluster. However the horizontal branch extends across the RR Lyrae gap into the blue region. Its horizontal branch index $\mathrm{HB}$, is about -0.85 , where $\mathrm{HB}=\mathrm{B}-\mathrm{R} / \mathrm{B}+\mathrm{V}+\mathrm{R}$, and $\mathrm{B}, \mathrm{V}, \mathrm{R}$, are the numbers of blue, variable and red horizontal branch stars. Clusters such as 47 Tuc with $[\mathrm{Fe} / \mathrm{H}]$ near -0.7 have $\mathrm{HB}$ values of -1.0 .

The period distribution of its RR Lyrae stars is most unusual. The mean period of the type $\mathrm{AB}$ stars is indicative of membership in the Oosterhof II category due to the fact that it also contains several variables with periods greater than 0.75 days and large amplitudes. Such variables are extremely rare in globular clusters and in the Galactic field.

Both the presence of blue horizontal branch stars and the presence of long period RR Lyrae stars in a metal-rich cluster require a new look at the parameters that are usually employed to reproduce the C-M diagram and other properties of the cluster. The usual suspects, age and metallicity, do not seem to be able to do it, unless there is a spread in one or both of them. To investigate the overall metallicity as well as the abundances of many elements we have initiated a program of high resolution spectroscopy of the red giants in NGC 6388. 
Table 1. Parameters of the program stars

\begin{tabular}{ccccccccc}
\hline Star & $\mathrm{V}$ & $\mathrm{B}-\mathrm{V}$ & $\mathrm{V}-\mathrm{R}$ & Number Spectra & $\mathrm{T}_{\text {eff }}$ & $\log g$ & $\mathrm{~V}_{t}$ & {$[\mathrm{Fe} / \mathrm{H}]$} \\
\hline 17 & 14.84 & 2.00 & 1.10 & 2 & 3600 & -0.15 & 1.4 & -0.89 \\
91 & 15.00 & 2.18 & 1.13 & 1 & 3500 & -0.30 & 1.6 & -0.85 \\
138 & 15.09 & 1.98 & 1.02 & 3 & 3850 & 0.00 & 1.3 & -0.78 \\
154 & 14.97 & 1.82 & 1.03 & 3 & 3800 & 0.00 & 1.4 & -0.75 \\
189 & 14.97 & 1.89 & 1.03 & 3 & 3800 & 0.00 & 1.4 & -0.74 \\
215 & 15.10 & 2.00 & 1.08 & 2 & 3700 & -0.25 & 1.4 & -0.79 \\
268 & 15.04 & 1.93 & 1.08 & 3 & 3700 & -0.25 & 1.5 & -0.86 \\
361 & 14.92 & 2.08 & 1.16 & 3 & 3550 & -0.25 & 1.5 & -0.62 \\
\hline
\end{tabular}

Table 2. Mean abundance expressed as $[\mathrm{X} / \mathrm{H}]$

\begin{tabular}{cccccc}
\hline Element & {$[\mathrm{X} / \mathrm{H}]$} & mean error & Element & {$[\mathrm{X} / \mathrm{H}]$} & mean error \\
\hline $\mathrm{Fe}$ & -0.8 & 0.03 & $\mathrm{Ca}$ & -0.8 & 0.06 \\
$\mathrm{C}$ & -1.4 & 0.06 & $\mathrm{Sc}$ & -0.85 & 0.05 \\
$\mathrm{O}$ & -0.9 & 0.06 & $\mathrm{Ti}$ & -0.7 & 0.06 \\
$\mathrm{Na}$ & -0.5 & 0.09 & $\mathrm{Alpha}$ & -0.65 & 0.09 \\
$\mathrm{Mg}$ & -0.5 & 0.06 & $\mathrm{Ba}, \mathrm{La}$ & -0.6 & 0.08 \\
$\mathrm{Al}$ & -0.5 & 0.10 & $\mathrm{Eu}$ & -0.6 & 0.08 \\
$\mathrm{Si}$ & -0.5 & 0.04 & & & \\
\hline
\end{tabular}

\section{Observations and analysis}

Spectra of resolving power of about 35,000 of 8 stars were aquired with the echelle spectrograph on the 4-m Blanco telescope at the CTIO. The S/N ratio peaked at about 100 near $7000 \AA$ and deteriorated to about 70 at the ends of the wavelength coverage.

We used solar $\log g f$ values so that all abundances are relative to the solar value. Effective temperatures were derived from the depths of neutral iron lines because the colors are affected by significant reddening which may vary across the cluster. Given the $T_{\text {eff }}$, the distance to the cluster of about $8 \mathrm{Kpc}$ and an estimate of the stellar masses of $0.7 \mathrm{M}_{\odot}$ the value of $\log g$ was derived. The basic parameters of the stars are shown in Table 1.

\section{Results}

Averaged abundances for 8 program stars are given in Table 2. The mean errors are internal only and do not include the affects of uncertainties in the stellar atmosphere.

\section{Discussion}

We have found no evidence for a spread in metallicity within the sample of stars selected for observation. The low $[\mathrm{O} / \mathrm{Fe}]$ value by about 0.3 dex as compared to most stars with a metallicity of -0.8 might be significant in the structure of the horizontal branch of NGC 6388. The reduced carbon as compared to oxygen is at the level to be expected for red giant tip stars. This indicates that carbon may have also been as deficient as oxygen in these stars when they were on the main sequence. The small excesses of sodium and aluminum may be indicative of proton capture activity. The mean alpha abundance shows about the normal excess for stars with $[\mathrm{Fe} / \mathrm{H}]$ near -0.8 . Both the $s$-process elements, barium and lanthanum as well the $r$-process element europium show small excesses which are well within the scatter seen among stars of small metal deficiencies. 